\title{
COMPLEX CYCLES AS OBSTRUCTIONS ON REAL ALGEBRAIC VARIETIES
}

\author{
WOJCIECH KUCHARZ \\ Institute of Mathematics, \\ Faculty of Mathematics and Computer Science, \\ Jagiellonian University, \\ ul. Profesora Lojasiewicza 6, \\ 30-348 Kraków, \\ Poland \\ E-mail: Wojciech.Kucharz@im.uj.edu.pl
}

(Received 5 April 2013; revised 24 September 2013; accepted 24 February 2014; first published online 19 December 2014)

\begin{abstract}
Let $Y$ be a compact nonsingular real algebraic variety of positive dimension. Then one can find a compact connected nonsingular real algebraic variety $X$, which admits a continuous map into $Y$ that is not homotopic to any regular map. It is hard to determine the minimum dimension of such a variety $X$. In this paper, new upper bounds for $\operatorname{dim} X$ are obtained. The main role in the constructions is played by complex algebraic cycles on $Y$.
\end{abstract}

\section{Mathematics Subject Classification. 14P05, 14P25}

1. Introduction. In the present paper, we investigate obstructions to representing homotopy classes of continuous maps, between real algebraic varieties, by regular maps. The term real algebraic variety designates a locally ringed space isomorphic to an algebraic subset of $\mathbb{R}^{N}$, for some $N$, endowed with the Zariski topology and the sheaf of real-valued regular functions (such an object is called an affine real algebraic variety in [3]). The class of real algebraic varieties is identical with the class of quasi-projective real algebraic varieties, cf. [3, Proposition 3.2.10, Theorem 3.4.4]. Morphisms of real algebraic varieties are called regular maps. Each real algebraic variety carries also the Euclidean topology, which is induced by the usual metric on $\mathbb{R}$. Unless explicitly stated otherwise, all topological notions relating to real algebraic varieties refer to the Euclidean topology.

In [6], a numerical invariant $\beta(Y)$ was defined for any real algebraic variety $Y$. Recall that $\beta(Y)$ is the supremum of all nonnegative integers $n$ with the following property: For every $n$-dimensional compact connected nonsingular real algebraic variety $X$, every continuous map from $X$ into $Y$ is homotopic to a regular map. Henceforth $Y$ will be assumed to be compact and nonsingular. For any nonnegative integer $k$, let $H_{\mathrm{alg}}^{k}(Y ; \mathbb{Z} / 2)$ denote the subgroup consisting of all algebraic cohomology classes in the cohomology group $H^{k}(Y ; \mathbb{Z} / 2)$, cf. [7] or [1, 3, 5]. According to [6, Theorem 2.9], $\beta(Y) \leq k$ if $H_{\mathrm{alg}}^{k}(Y ; \mathbb{Z} / 2) \neq 0$ for some $k \geq 1$. As a consequence, one gets some upper bounds for $\beta(Y)$, which are independent of the algebraic-geometric structure of $Y$. For example, $\beta(Y) \leq \operatorname{dim} Y$ if $\operatorname{dim} Y \geq 1$. This assertion holds since $H_{\text {alg }}^{d}(Y ; \mathbb{Z} / 2)=H^{d}(Y ; \mathbb{Z} / 2) \neq 0$ for $d=\operatorname{dim} Y$. Furthermore, $\beta(Y) \leq k$ if the $k$ th Stiefel-Whitney class $w_{k}(Y)$ of $Y$ is nonzero for some $k \geq 1$. Indeed, it suffices to note that $w_{k}(Y)$ is in $H_{\text {alg }}^{k}(Y ; \mathbb{Z} / 2)$, cf. [7] or [1]. In particular, $\beta(Y) \leq 1$ if $Y$ 
is nonorientable (as a smooth, that is, $\mathcal{C}^{\infty}$ manifold), the orientability of $Y$ being equivalent to $w_{1}(Y)=0$.

The reader may consult [9] for other results related to the problem under consideration.

In this paper, new upper bounds for $\beta(Y)$ are obtained. For $Y$ orientable they are frequently sharper than those given in [6]. The key role is played by $\mathbb{C}$-algebraic cohomology classes. Let $H_{\mathbb{C} \text {-alg }}^{2 k}(Y ; \mathbb{Z})$ denote the subgroup consisting of all $\mathbb{C}$-algebraic cohomology classes in the cohomology group $H^{2 k}(Y ; \mathbb{Z})$, cf. [2]. For the convenience of the reader, the definition of $H_{\mathbb{C} \text {-alg }}^{2 k}(Y ; \mathbb{Z})$ is recalled in Section 2.

THEOREM 1.1. Let $Y$ be a compact nonsingular real algebraic variety. If the group $H_{\mathbb{C}-\text { alg }}^{2 k}(Y ; \mathbb{Z})$ is infinite for some $k \geq 1$, then $\beta(Y) \leq 2 k-1$.

The proof is postponed until Section 2. It is worthwhile to record two consequences of Theorem 1.1. Let $p_{k}(Y) \in H^{4 k}(Y ; \mathbb{Z})$ denote the $k$ th Pontryagin class of $Y$.

COROLLARY 1.2. Let $Y$ be a compact nonsingular real algebraic variety. If for some integer $k \geq 1$, the Pontryagin class $p_{k}(Y)$ is an element of infinite order in the group $H^{4 k}(Y ; \mathbb{Z})$, then $\beta(Y) \leq 4 k-1$.

Proof. In view of [2, Theorem 5.3], $p_{k}(Y)$ is in $H_{\mathbb{C} \text {-alg }}^{4 k}(Y ; \mathbb{Z})$, and hence it suffices to apply Theorem 1.1.

A compact oriented smooth manifold is said to be an oriented boundary if it is the boundary, with induced orientation, of a compact oriented smooth manifold with boundary.

COROLlaRY 1.3. Let $Y$ be a compact nonsingular real algebraic variety. If $Y$ is oriented, and the disjoint union of two copies of $Y$ is not an oriented boundary, then $\beta(Y) \leq \operatorname{dim} Y-1$.

Proof. Let $M$ be the disjoint union of two copies of $Y$. Then $M$ is the unoriented boundary of $Y \times[0,1]$, and hence its Stiefel - Whitney numbers are all equal to 0 . Since $M$ is not an oriented boundary, it follows that at least one Pontryagin number of $M$ is different from 0 (cf. Wall's refinement [14] of earlier results due to Thom [13] and other authors). Consequently, $\operatorname{dim} Y=4 d$, for some positive integer $d$, and there exists an element of infinite order in $H^{4 d}(Y ; \mathbb{Z})$, which is a polynomial in the Pontryagin classes $p_{k}(Y)$ for $k \geq 0$. In particular, $p_{k}(Y)$ is an element of infinite order in the group $H^{4 k}(Y ; \mathbb{Z})$ for some $k \geq 1$. Now it suffices to apply Corollary 1.2.

2. Complex algebraic cycles. First some basic definitions will be recalled. Let $V$ be a nonsingular projective complex algebraic variety. For any nonnegative integer $k$, a cohomology class in $H^{2 k}(V ; \mathbb{Z})$ is said to be algebraic if it corresponds via the cycle map to an algebraic cycle of codimension $k$ on $V$, cf. [8, Chapter 19]. The set $H_{\text {alg }}^{2 k}(V ; \mathbb{Z})$ of all algebraic cohomology classes in $H^{2 k}(V ; \mathbb{Z})$ forms a subgroup. This construction can be transferred in a suitable way to the real algebraic-geometric setting.

Let $X$ be a compact nonsingular real algebraic variety. A nonsingular projective complexification of $X$ is a pair $(W, j)$, where $W$ is a nonsingular projective complex algebraic variety defined over $\mathbb{R}$ and $j: X \rightarrow W$ is an injective map, such that $W(\mathbb{R})$ is Zariski dense in $W, j(X)=W(\mathbb{R})$ and $j$ induces a biregular isomorphism between $X$ and $W(\mathbb{R})$. Here $W(\mathbb{R})$ denotes the set of real points of $W$. The existence of $(W, j)$ 
follows from Hironaka's theorem on resolution of singularities $[\mathbf{1 0}, \mathbf{1 1}]$. The subgroup

$$
H_{\mathbb{C} \text {-alg }}^{2 k}(X ; \mathbb{Z}):=j^{*}\left(H_{\mathrm{alg}}^{2 k}(W ; \mathbb{Z})\right)
$$

of $H^{2 k}(X ; \mathbb{Z})$ does not depend on the choice of $(W, j)$. Any cohomology class in $H_{\mathbb{C} \text {-alg }}^{2 k}(X ; \mathbb{Z})$ is said to be $\mathbb{C}$-algebraic. The groups $H_{\mathbb{C} \text {-alg }}^{2 k}(-; \mathbb{Z})$ have the expected functorial property: If $f: X \rightarrow Y$ is a regular map between compact nonsingular real algebraic varieties, then

$$
f^{*}\left(H_{\mathbb{C} \text {-alg }}^{2 k}(Y ; \mathbb{Z})\right) \subseteq H_{\mathbb{C} \text {-alg }}^{2 k}(X ; \mathbb{Z}) .
$$

These properties of $H_{\mathbb{C} \text {-alg }}^{2 k}(-; \mathbb{Z})$ are proved in [2].

In order to interpret $\mathbb{C}$-algebraic cohomology classes as obstructions, one needs a somewhat refined version of Thom's representability theorem [13, Théorème III.4]. For any $n$-dimensional compact oriented smooth manifold $N$, let $[N]$ denote its fundamental class in $H_{n}(N ; \mathbb{Z})$.

THEOREM 2.1. Let $Y$ be a $C W$-complex and let $\alpha$ be a homology class in $H_{n}(Y ; \mathbb{Z})$, with $n \geq 1$. Then there exist an $n$-dimensional compact oriented smooth manifold $N$, a continuous map $f: N \rightarrow Y$ and a positive integer $l$ such that $f_{*}([N])=l \alpha$ and $N$ is an oriented boundary. Furthermore, if $\alpha$ is represented by a singular cycle with support contained in a connected component of $Y$, then the manifold $N$ can be chosen connected.

Proof. One can assume without loss of generality that $Y$ is compact and connected. The argument used in [13, pp. 57, 58] implies the existence of a compact connected orientable smooth manifold $P$ containing $Y$ as a retract. One can find such a manifold $P$ with $p:=\operatorname{dim} P \geq 2 n+1$. Let $i: Y \hookrightarrow P$ be the inclusion map and let $r: P \rightarrow Y$ be a retraction. According to Thom's theorem [13, Théorème II.29], there exists a positive integer $l$ such that the homology class $l_{*}(\alpha)$ in $H_{n}(P ; \mathbb{Z})$ can be represented by an $n$-dimensional compact oriented smooth submanifold $M$ of $P$. Since the manifold $P$ is connected and $1 \leq n \leq p-2$, the connected components of $M$ can be joined with $n$-dimensional tubes in $P$. Hence $M$ can be assumed to be connected. Let $U$ be an open subset of $P \backslash M$ that is diffeomorphic to $\mathbb{R}^{p}$. Since $p \geq 2 n+1$, there exists a smooth submanifold $M^{\prime}$ of $U$, diffeomorphic to $M$. Choosing an orientation of $M^{\prime}$ and joining the submanifolds $M$ and $M^{\prime}$ with an $n$-dimensional tube in $P$, one obtains a compact connected oriented smooth submanifold $N$ of $P$ representing the homology class $l i_{*}(\alpha)$. Furthermore, if the orientation of $M^{\prime}$ is suitably chosen, then $N$ is diffeomorphic to the connected sum $M \#(-M)$, where $-M$ stands for $M$ with the opposite orientation. Since $M \#(-M)$ represents the same oriented bordism class as the disjoint union of $M$ and $-M$, it follows that $N$ is an oriented boundary. If $j: N \hookrightarrow P$ is the inclusion map and $f:=r \circ j$, then $j_{*}([N])=l i_{*}(\alpha)$ and

$$
f_{*}([N])=r_{*}\left(j_{*}([N])\right)=l r_{*}\left(i_{*}(\alpha)\right)=l(r \circ i)_{*}(\alpha)=l \alpha .
$$

The proof is complete.

Proof of Theorem 1.1. Let $u$ be an element of infinite order in the group $H_{\mathbb{C} \text {-alg }}^{2 k}(Y ; \mathbb{Z})$. Then there exists a homology class $\alpha$ in $H_{2 k}(Y ; \mathbb{Z})$ such that the Kronecker index $\langle u, \alpha\rangle$ is different from 0 . One can choose $\alpha$ in such a way that it is represented by a singular cycle with support contained in a connected component of $Y$. By Theorem 2.1, one can find a $2 k$-dimensional compact connected oriented smooth manifold $N$, a continuous 
map $f: N \rightarrow Y$ and a positive integer $l$ such that $f_{*}([N])=l \alpha$ and $N$ is an oriented boundary. The last named property of $N$ is crucial. According to [4, Theorem 3.3], it implies the existence of a nonsingular real algebraic variety $X$, which is diffeomorphic to $N$ and satisfies $H_{\mathbb{C} \text {-alg }}^{2 k}(X ; \mathbb{Z})=0$. If $\varphi: X \rightarrow N$ is a smooth diffeomorphism and $g:=f \circ \varphi$, then

$$
g_{*}([X])=f_{*}(\varphi([X]))=f_{*}([N])=l \alpha .
$$

Consequently,

$$
\left\langle g^{*}(u),[X]\right\rangle=\left\langle u, g_{*}([X])\right\rangle=\langle u, l \alpha\rangle=l\langle u, \alpha\rangle \neq 0,
$$

and hence $g^{*}(u) \neq 0$ in $H^{2 k}(X ; \mathbb{Z})$. Since $H_{\mathbb{C} \text {-alg }}^{2 k}(X ; \mathbb{Z})=0$, the functoriality of $H_{\mathbb{C} \text {-alg }}^{2 k}(-; \mathbb{Z})$ implies that the map $g$ is not homotopic to any regular map. Thus, $\beta(Y) \leq 2 k-1$, as required.

Any projective complex algebraic variety $V$ can be regarded as a real algebraic variety, denoted $V_{\mathbb{R}}$ (identify $\mathbb{C}$ with $\mathbb{R}^{2}$ ). Obviously, $\operatorname{dim}_{\mathbb{R}} V_{\mathbb{R}}=2 \operatorname{dim}_{\mathbb{C}} V$. Furthermore, $V$ and $V_{\mathbb{R}}$ coincide as topological spaces endowed with the Euclidean topology.

EXAMPLE 2.2. If $V$ is a nonsingular projective complex algebraic variety of positive dimension, then $\beta\left(V_{\mathbb{R}}\right) \leq 1$. Indeed, there are two subgroups, $H_{\text {alg }}^{2 k}(V ; \mathbb{Z})$ and $H_{\mathbb{C} \text {-alg }}^{2 k}\left(V_{\mathbb{R}} ; \mathbb{Z}\right)$, of the cohomology group $H^{2 k}\left(V_{\mathbb{R}} ; \mathbb{Z}\right)$. It is well known that $H_{\text {alg }}^{2 k}(V ; \mathbb{Z}) \subseteq H_{\mathbb{C} \text {-alg }}^{2 k}\left(V_{\mathbb{R}}\right)$, cf. [12]. Since the group $H_{\text {alg }}^{2}(V ; \mathbb{Z})$ is infinite, the inequality $\beta\left(V_{\mathbb{R}}\right) \leq 1$ follows from Theorem 1.1. Consequently, if $V$ is connected and simply connected, then $\beta\left(V_{\mathbb{R}}\right)=1$. It does not seem that these facts can be established using methods developed in [6].

ACKNOWLEDGEMENT. The author was partially supported by NCN grant 2011/01/B/ST1/01289.

\section{REFERENCES}

1. R. Benedetti and A. Tognoli, Remarks and counterexamples in the theory of real vector bundles and cycles, in Géométrie algebraique réelle et formes quadratiques, Lecture Notes in Mathematics, vol. 959 (Springer, 1982), 198-211.

2. J. Bochnak, M. Buchner and W. Kucharz, Vector bundles over real algebraic varieties, K-Theory 3 (1989), 271-298.

J. Bochnak, M. Buchner and W. Kucharz, Vector bundles over real algebraic varieties, Erratum: K-Theory 4 (1990), 103.

3. J. Bochnak, M. Coste and M.-F. Roy, Real algebraic geometry, Ergeb. Math. Grenzgeb., vol. 36 (Berlin, Springer, 1998).

4. J. Bochnak and W. Kucharz, On real algebraic morphisms into even-dimensional spheres, Ann. Math. 128 (1988), 415-433.

5. J. Bochnak and W. Kucharz, On homology classes represented by real algebraic varieties, in Singularities Symposium - Łojasiewicz 70, Banach Center Publ. 44, Inst. Math. Polish Acad. Sci. 21-35 (1998).

6. J. Bochnak and W. Kucharz, Real algebraic morphisms represent few homotopy classes, Math. Ann. 337 (2007), 909-921.

7. A. Borel and A. Haefliger, La classe d'homologie fondamentale d'un espace analytique, Bull. Soc. Math. France 89 (1961), 461-513. 
8. W. Fulton, Intersection theory, Ergeb. Math. Grenzgeb., vol. 2 (Berlin, Springer), 1984.

9. R. Ghiloni, Second order homological obstructions on real algebraic manifolds, Topol. Appl. 154 (2007), 3090-3094.

10. H. Hironaka, Resolution of singularities of an algebraic variety over a field of characteristic zero, Ann. Math. 79 (1964), 109-326.

11. J. Kollár, Lectures on resolutuon of singularities, Annals of Mathematics Studies, vol. 166 (Princeton University Press, Princeton, NJ, 2007).

12. W. Kucharz, Algebraic cycles and realification of complex projecive varieties, Geom. Dedicata 54 (1995), 317-322.

13. R. Thom, Quelques propriétiés globales des variétés différentiables, Comment. Math. Helv. 28 (1954), 17-86.

14. C. T. C. Wall, Determinations of the cobordism ring, Ann. Math. 72 (1960), 292-311. 